\title{
Analysis of a native whitefly transcriptome and its sequence divergence with two invasive whitefly species
}

Xiao-Wei Wang ${ }^{1 *+}$, Qiong-Yi Zhao ${ }^{2 \dagger}$, Jun-Bo Luan ${ }^{1}$, Yu-Jun Wang ${ }^{1}$, Gen-Hong Yan ${ }^{1}$ and Shu-Sheng Liu ${ }^{1 *}$

\begin{abstract}
Background: Genomic divergence between invasive and native species may provide insight into the molecular basis underlying specific characteristics that drive the invasion and displacement of closely related species. In this study, we sequenced the transcriptome of an indigenous species, Asia II 3, of the Bemisia tabaci complex and compared its genetic divergence with the transcriptomes of two invasive whiteflies species, Middle East Asia Minor 1 (MEAM1) and Mediterranean (MED), respectively.

Results: More than 16 million reads of 74 base pairs in length were obtained for the Asia II 3 species using the Illumina sequencing platform. These reads were assembled into 52,535 distinct sequences (mean size: 466 bp) and 16,596 sequences were annotated with an E-value above $10^{-5}$. Protein family comparisons revealed obvious diversification among the transcriptomes of these species suggesting species-specific adaptations during whitefly evolution. On the contrary, substantial conservation of the whitefly transcriptomes was also evident, despite their differences. The overall divergence of coding sequences between the orthologous gene pairs of Asia II 3 and MEAM1 is $1.73 \%$, which is comparable to the average divergence of Asia II 3 and MED transcriptomes (1.84\%) and much higher than that of MEAM1 and MED (0.83\%). This is consistent with the previous phylogenetic analyses and crossing experiments suggesting these are distinct species. We also identified hundreds of highly diverged genes and compiled sequence identify data into gene functional groups and found the most divergent gene classes are Cytochrome P450, Glutathione metabolism and Oxidative phosphorylation. These results strongly suggest that the divergence of genes related to metabolism might be the driving force of the MEAM1 and Asia II 3 differentiation. We also analyzed single nucleotide polymorphisms within the orthologous gene pairs of indigenous and invasive whiteflies which are helpful for the investigation of association between allelic and phenotypes.
\end{abstract}

Conclusions: Our data present the most comprehensive sequences for the indigenous whitefly species Asia II 3. The extensive comparisons of Asia II 3, MEAM1 and MED transcriptomes will serve as an invaluable resource for revealing the genetic basis of whitefly invasion and the molecular mechanisms underlying their biological differences.

Keywords: Bemisia tabaci, Biological invasion, Genetic divergence, Indigenous species, Next generation sequencing, Transcriptome, Whitefly

\footnotetext{
* Correspondence: xwwang@zju.edu.cn; shshliu@zju.edu.cn

${ }^{+}$Equal contributors

${ }^{1}$ Ministry of Agriculture Key Laboratory of Agricultural Entomology, Institute

of Insect Sciences, Zhejiang University, 866 Yuhangtang Road, Hangzhou

310058, China

Full list of author information is available at the end of the article
}

\section{Biomed Central}

(C) 2012 Wang et al.; licensee BioMed Central Ltd. This is an Open Access article distributed under the terms of the Creative Commons Attribution License (http://creativecommons.org/licenses/by/2.0), which permits unrestricted use, distribution, and reproduction in any medium, provided the original work is properly cited. 


\section{Background}

The whitefly Bemisia tabaci (Gennadius) (Hemiptera: Aleyrodidae) is a species complex composed of at least 31 morphologically indistinguishable cryptic species (hereafter referred to as "species") [1-6]. These species differ genetically as well as in host range, fecundity, insecticide resistance, mating behavior and ability to transmit begomoviruses [7-12]. While many species within the B. tabaci complex cause no obvious harms to agricultural production; some members of this species complex are highly invasive and cause extensive damage to agricultural, horticultural, and ornamental crops through direct feeding or the transmission of plant viruses $[13,14]$. Two species of the B. tabaci complex, Middle East - Asia Minor 1 (previously known as biotype B; hereafter MEAM1) and Mediterranean (previously known as biotype Q; hereafter MED) have risen to international prominence due to their global invasion during the last 20 years $[8,15]$. MEAM1 and MED originated from the Middle East Asia Minor and Mediterranean Basin regions respectively, and have invaded many countries around the world [3,16]. Extensive evidence has indicated that the invasion of MEAM1 and MED are associated with the displacement of their closely related indigenous whitefly species $[8,14]$.

The invasion of an alien whitefly species and competition between invasive and indigenous species are mediated by many abiotic and biotic factors. Efforts have been made to understand the factors that contribute to the incursion of the two species into new regions and the displacement of indigenous species. For example, the invasion of MEAM1 is assumed to be associated with its high adaptability under various environmental stresses and host plants $[9,10,17,18]$. Liu et al. [8] also revealed that the displacement of indigenous whitefly species by MEAM1 is associated with the behavior of mating interference. On the other hand, the spread of MED is closely related to its ability to maintain high levels of resistance to major classes of insecticides [19-22]. Despite these advances, the molecular mechanisms underlying the extraordinary capacity of MEAM1 and MED to spread and ultimately displace the native species remains largely unknown. Furthermore, previous studies have mainly focused on single gene or individual aspect of the B. tabaci biology, a global picture of the genetic factors associated with the invasion of these two whitefly species is still lacking.

The genomic divergence between invasive and indigenous species is valuable for determining how phenotypes specific to invasive species have been formed [23]. By examining the divergence of large numbers of genes, a overall picture of genetic differences and invasion mechanisms may be attained [24]. Here, we propose that a global analysis of genomic divergence among the B. tabaci species complex will reveal the molecular mechanisms underlying the biological invasions of MEAM1 and MED. First, the $B$. tabaci species are reproductively isolated, but retain sufficient genetic similarity for comparative analyses $[4,25,26]$. Second, the whitefly species went through an allopatric divergence process and showed significant differences in survival and reproductive performance $[16,27,28]$. This warrants exploring the interspecies evolutionary processes through the comparison of orthologous genes. Third, at least 31 species have been delineated for the B. tabaci complex including 2 invasive species and 29 indigenous species. The rich diversity of invasive and indigenous species allows extensive cross comparisons of orthologous genes among difference members of the complex, which will facilitate the elucidation of invasive mechanisms.

The transcriptomes of two invasive whitefly species MED and MEAM1 have been sequenced using Illumina sequencing technology $[29,30]$. In this study, we sequenced the transcriptome of an indigenous $B$. tabaci species - Asia II 3 (previously known as biotype ZHJ1) and generated 52,535 distinct sequences. These transcriptome sequences provide a rich molecular resource for functional analysis of the native $B$. tabaci species. In order to gain further insights on how genes have diverged between the indigenous and invasive whiteflies, we compared the global sequence divergence between the transcriptomes of Asia II 3 and the invasive species MEAM1 and MED. The identification and analysis of divergent sequences between the indigenous and invasive whitefly species opens the door for future investigations on the molecular mechanisms of $B$. tabaci invasion. The approach described in this manuscript will significantly accelerate the identification of genetic variation underlying adaptation in B. tabaci and other invasive species.

\section{Results and discussion}

Illumina sequencing and assembly of Asia II 3 transcriptome

Using Illumina technology, the transcriptome of the indigenous Asia II 3 whitefly species was sequenced. A total of $16,871,140$ reads of 74 base pairs long were obtained in a single run and were assembled into 144,103 contigs (average length: $201 \mathrm{bp}$ ) with SOAPdenovo software [31]. The contigs were assembled into 77,263 scaffolds (mean size: $359 \mathrm{bp}$ ) and further clustered into 52,535 distinct sequences (mean size: $466 \mathrm{bp}$ ) (Table 1). Overall, these results are comparable to the transcriptome of the invasive MEAM1 which contains 104,722 scaffolds (mean size: 326 bp) and 57,741 distinct sequences with an average length of 479 bp (Additional file 1) [30]. However, compared to the transcriptome of MED, which contains 168,900 distinct sequences, the number of genes in the Asia II 3 transcriptome is much less. This is probably due to the lower number of sequencing reads in the transcriptomes of Asia II 3 (16.8 
Table 1 Summary for the Asia II 3 B. tabaci transcriptome

\begin{tabular}{ll}
\hline Total number of reads & $16,871,140$ \\
\hline Total base pairs (bp) & $1,248,464,360$ \\
\hline Average read length (bp) & 74 \\
\hline Total number of contigs & 144,103 \\
\hline Mean length of contigs ( bp) & 201 \\
\hline Total number of scaffolds & 77,263 \\
\hline Mean length of scaffolds (bp) & 359 \\
\hline Distinct sequences & 52,535 \\
\hline Sequences with E-value $<10^{-5}$ & 15,357
\end{tabular}

million) and MEAM1 (17 million) compared to the MED transcriptome (43.7 million) (Additional file 1) $[29,30]$. Even though a large number of genes $(52,535)$ were identified, a large fraction of lowly expressed transcripts were probably missed due to a low number of sequence reads (16.8 million).

\section{Functional annotation of Asia II 3 transcriptome}

For functional annotation, distinct sequences were searched against the non-redundant (nr) NCBI nucleotide database and a total of 16,596 genes returned an above cut-off BLAST result representing about $31.6 \%$ of all distinct sequences (Additional file 2). This proportion is similar to the $20 \%$ to $40 \%$ of annotated sequences from a traditional Sanger sequenced EST library [32]. To determine the possible functions of assembled Asia II 3 genes, Gene Ontology (GO) assignments were used to classify the distinct sequences. Based on sequence homology, 4,819 sequences could be categorized into functional groups under the "Molecular function", "Biological process" and "Cellular component" divisions (Additional file 3). The functions of genes cover various biological processes and genes participate in "Cellular process" and "Metabolic process" are the most highly represented. Next, we compared the GO classification of the Asia II 3 transcriptome with that of MEAM1 and MED transcriptomes, respectively $[29,30]$ and found that the distributions of gene functions from these three species are similar (Additional file 3). These results suggest: i) the functions of genes from Asia II 3, MEAM1 and MED are highly conserved; ii) there is no bias in the construction of the libraries from these $B$. tabaci species.

\section{Analysis of Asia II 3 gene expression}

The level of Asia II 3 gene expression was analyzed based on the number of Reads Per Kilobase per Million mapped reads (RPKM); and a list of all the genes and

Table 2 Highly expressed genes in the transcriptome of Asia II 3

\begin{tabular}{|c|c|c|c|}
\hline Gene ID & Number of reads ${ }^{a}$ & RPKM $^{b}$ & Swissprot annotation \\
\hline BT_ZHJ1_ZJU_Unigene4448 & 13476 & 3433.5 & Actin-5, muscle-specific \\
\hline BT_ZHJ1_ZJU_Unigene5935 & 8392 & 3212.7 & Tubulin alpha-1 chain \\
\hline BT_ZHJ1_ZJU_Unigene44594 & 6567 & 2854.2 & $60 S$ ribosomal protein L18a \\
\hline BT_ZHJ1_ZJU_Unigene40759 & 4952 & 2613.5 & ADP,ATP carrier protein 2 \\
\hline BT_ZHJ1_ZJU_Unigene33425 & 3191 & 2213.8 & Elongation factor 1-alpha 2 \\
\hline BT_ZHJ1_ZJU_Unigene36010 & 3122 & 1946.6 & Glyceraldehyde-3-phosphate dehydrogenase \\
\hline BT_ZHJ1_ZJU_Unigene40277 & 3388 & 1820.6 & ATP synthase subunit beta, mitochondrial \\
\hline BT_ZHJ1_ZJU_Unigene33344 & 2299 & 1595.0 & Troponin T, skeletal muscle \\
\hline BT_ZHJ1_ZJU_Unigene42468 & 3239 & 1584.9 & Elongation factor 1-alpha \\
\hline BT_ZHJ1_ZJU_Unigene38168 & 2689 & 1552.2 & $40 S$ ribosomal protein $\mathrm{S} 12$ \\
\hline BT_ZHJ1_ZJU_Unigene44051 & 3336 & 1498.4 & Vitellogenin-A1 \\
\hline BT_ZHJ1_ZJU_Unigene43506 & 3232 & 1497.2 & $40 S$ ribosomal protein S11 \\
\hline BT_ZHJ1_ZJU_Unigene49876 & 5853 & 1473.5 & $60 S$ ribosomal protein L15 \\
\hline BT_ZHJ1_ZJU_Unigene43457 & 3079 & 1430.8 & $40 S$ ribosomal protein $\$ 20$ \\
\hline BT_ZHJ1_ZJU_Unigene46015 & 3456 & 1358.3 & 605 ribosomal protein $\mathrm{L} 6$ \\
\hline BT_ZHJ1_ZJU_Unigene37661 & 2284 & 1344.7 & Ferritin, middle subunit \\
\hline BT_ZHJ1_ZJU_Unigene38243 & 2286 & 1319.6 & Paramyosin, short form \\
\hline BT_ZHJ1_ZJU_Unigene45492 & 3074 & 1258.3 & ATP synthase subunit alpha, mitochondrial \\
\hline BT_ZHJ1_ZJU_Unigene44482 & 2871 & 1255.2 & Vitellogenin \\
\hline BT_ZHJ1_ZJU_Unigene34665 & 1906 & 1251.8 & 40 S ribosomal protein $\mathrm{S} 2$ \\
\hline
\end{tabular}

${ }^{\mathrm{a}}$ The total number of reads mapped to each gene.

${ }^{b}$ Gene expression level was determined by calculating the number of reads for each gene and then normalizing to RPKM (reads per kilobase per million mapped reads). 
expression levels are shown in Additional file 4. To our knowledge, this is the first global analysis of gene expression level in B. tabaci. Twenty annotated genes with very high expression levels (RPKM>1,250) were found. Many of the genes with significant expression levels were involved in cell structure (e.g. actin and tubulin), ribosome (e.g. 60S ribosomal protein, 40S ribosomal protein) and energy metabolism (e.g. ATP synthase and glyceraldehyde-3-phosphate dehydrogenase) (Table 2). This finding is not surprising, as these genes are essential for the survival of an organism. Next, we grouped Asia II 3 genes into three categories based on their levels of expression. Roughly, $14 \%$ of the genes were highly expressed (RPKM> 50), $25 \%$ of the transcripts were in medium level $(50>$ RPKM $>20)$ and $61 \%$ of the genes had a $\mathrm{RPKM}<20$ (Figure $1 \mathrm{~A}$ ). Conversely, when calculating the number of reads for each gene, it was found that the small fraction of highly expressed genes constituted approximately $61 \%$ of the sequenced reads, whereas only $13 \%$ of the reads were from the genes with low expression levels (Figure 1B).

\section{Identification and analysis of $B$. tabaci protein families}

To compare the transcriptomes of Asia II 3, MEAM1 and MED, protein families for the three species were built. A total of 10,217 protein families were identified based on sequence similarities (Figure 2). The number of protein families for MED transcriptome was 8,405, which is much higher than that of Asia II $3(4,938)$ and MEAM1 (6,174). In this comparison, both diversification and conservation of the $B$. tabaci transcriptomes were obvious at the protein family level. A total of 3,023 protein families were conserved among the transcriptomes of Asia II 3, MEAM1 and MED which represent the core cellular and physiological proteins (hereafter "Core protein families") common to the three species. The total number of protein families found in only one species was 3,940 (2,756+582+602), which might be responsible for the differences and unique features of each species (Figure 2). However, as these transcriptomes are incomplete, the number of species-specific protein families is likely to change when more genes are sequenced. Among these specific protein families, the majority was from MED (2,756). This is probably due to the higher sequencing amount of MED (3G) compared to Asia II 3 (1G) and MEAM1 (1G) (Additional file 1) [29,30]. To reveal the common characteristics of the whitefly transcriptomes, a hypergeometric test was implemented to identify enriched GO terms in the "Core protein families". A total of 18 protein families were significantly enriched (Table 3). Interestingly, 3 of the 18 enriched GO terms were related to nucleotide binding. This phenomenon is consistent with the previous finding in the analysis of nematode transcriptomes [33] and

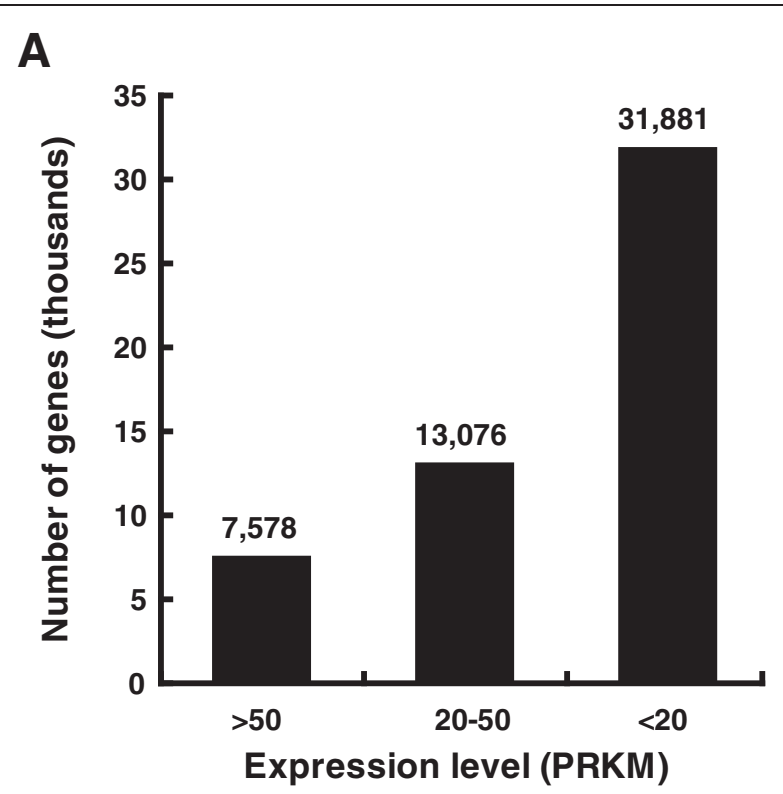

B

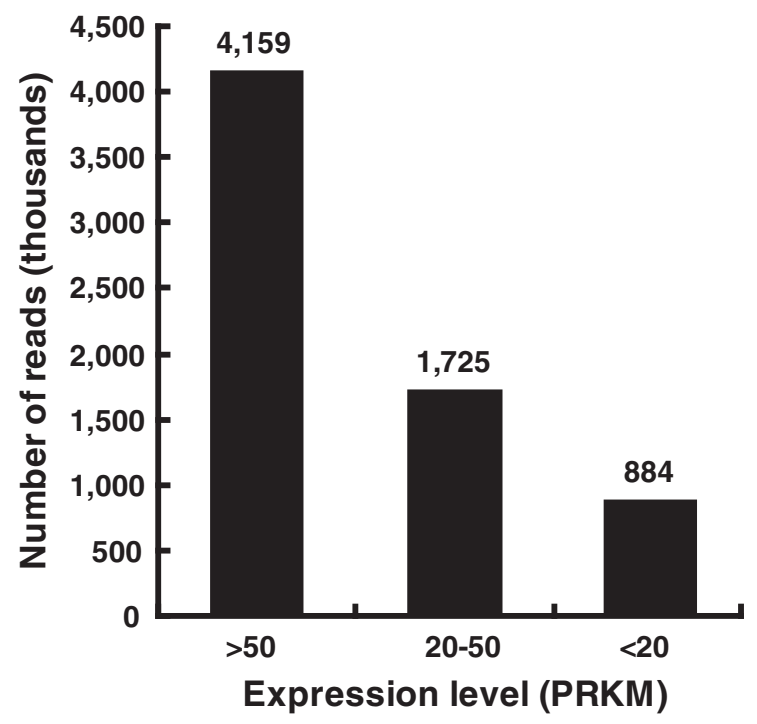

Figure 1 Levels of gene expression of Asia II 3 transcriptome. A. Percentages of Asia II 3 genes that belong to high (RPKM $>50$ ), medium $(50>$ RPKM $>20)$ and low $($ RPKM $<20)$ expression levels based on the total number of Reads Per Kilobase per Million mapped reads (RPKM). B. Numbers of reads mapped to the genes of high $($ RPKM $>50)$, medium $(50>$ RPKM $>20)$ and low $($ RPKM $<20)$ expression levels. The exact values are shown on the top of each bar.

demonstrates the importance and conservation of nucleotide binding proteins in different species. The other major GO terms enriched in the "Core protein families" were related to amino acid transporter, protein folding, proteolysis and peptidase, suggesting the critical roles of protein transportation and metabolism among the three B. tabaci species (Table 3 ). 


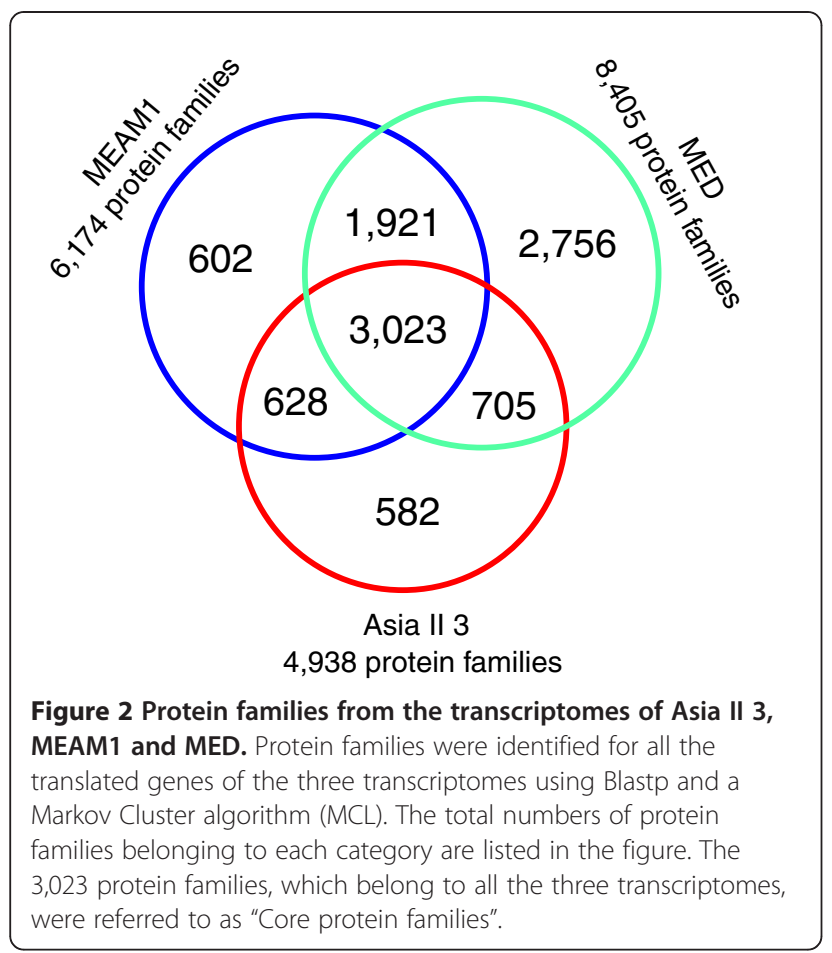

Identification of the orthologous genes between Asia II 3 and MEAM1, Asia II 3 and MED

Possible orthologous genes between the transcriptomes of Asia II 3 and MEAM1were identified according to the diagram in Figure 3 (left). Between Asia II 3 and MEAM1, a total of 20,929 pairs of possible orthologs were identified. Among these sequence pairs, 3,308 pairs of sequences could be unambiguously mapped to the same protein in Swissprot database, suggesting strongly that they are orthologous genes (Figure 3). Based on the coding information of Swissprot hits, the untranslated region (UTR) of each gene pair was predicted (210 pairs of 5'UTR and 337 pairs of 3'UTR) and 2,966 pairs of orthologous coding sequences (CDS) were obtained (Figure 3 and Additional file 5). The features of the 2,966 orthologous genes are listed in Table 4. Similarly, 22,415 pairs of potential orthologs were identified between Asia II 3 and MED (Figure 3, right). After the Swissprot annotation, 102 pairs of 5'UTRs, 102 pairs of 3' UTRs and 2,529 pairs of CDS were identified between the transcriptomes of Asia II 3 and MED (Table 4). The average divergence for the 2,966 orthologous genes between Asia II 3 and MEAM1 is $1.73 \%$. This difference is comparable to the average divergence between Asia II 3 and MED (1.84\%) and much higher than the divergence

Table 3 Statistically enriched Gene Ontology terms in the "Core protein families"

\begin{tabular}{|c|c|c|c|c|c|c|c|}
\hline \multirow[t]{2}{*}{ GO term } & \multicolumn{4}{|c|}{$\begin{array}{l}\text { Number of } \\
\text { Core protein } \\
\text { family genes } \\
\text { mapped to } \\
\text { each GO }\end{array}$} & \multirow[t]{2}{*}{$\begin{array}{c}\text { All } \\
\text { transcriptome } \\
\text { genes } \\
\text { mapped to } \\
\text { each GO }\end{array}$} & \multirow[t]{2}{*}{$p$-value } & \multirow[t]{2}{*}{ GO annotation } \\
\hline & MEAM1 & MED & Asia II 3 & Total & & & \\
\hline GO:0005524 & 264 & 222 & 243 & 729 & 920 & $1.60 \mathrm{E}-16$ & ATP binding \\
\hline GO:0005525 & 70 & 55 & 48 & 173 & 202 & $2.55 \mathrm{E}-09$ & GTP binding \\
\hline GO:0003924 & 45 & 29 & 31 & 105 & 119 & $1.34 \mathrm{E}-07$ & GTPase activity \\
\hline GO:0005811 & 72 & 51 & 64 & 187 & 230 & $1.82 \mathrm{E}-06$ & lipid particle \\
\hline GO:0005198 & 18 & 19 & 15 & 52 & 56 & $6.42 \mathrm{E}-06$ & structural molecule activity \\
\hline GO:0006865 & 11 & 18 & 13 & 42 & 44 & $7.50 \mathrm{E}-06$ & amino acid transport \\
\hline GO:0006457 & 23 & 14 & 27 & 64 & 71 & $7.68 \mathrm{E}-06$ & protein folding \\
\hline GO:0008152 & 77 & 85 & 50 & 212 & 267 & 9.75E-06 & metabolic process \\
\hline GO:0015171 & 8 & 17 & 9 & 34 & 35 & $1.91 \mathrm{E}-05$ & amino acid transmembrane transporter activity \\
\hline GO:0051301 & 15 & 14 & 15 & 44 & 47 & 1.99E-05 & cell division \\
\hline GO:0005215 & 17 & 18 & 24 & 59 & 66 & $3.30 \mathrm{E}-05$ & transporter activity \\
\hline GO:0004252 & 24 & 20 & 15 & 59 & 66 & $3.30 \mathrm{E}-05$ & serine-type endopeptidase \\
\hline GO:0030126 & 9 & 7 & 10 & 26 & 26 & 3.69E-05 & COPI vesicle coat \\
\hline GO:0006508 & 49 & 52 & 38 & 139 & 171 & $3.90 \mathrm{E}-05$ & proteolysis \\
\hline GO:0000166 & 81 & 55 & 60 & 196 & 249 & 5.65E-05 & nucleotide binding \\
\hline GO:0005506 & 47 & 24 & 20 & 91 & 108 & $6.35 \mathrm{E}-05$ & iron ion binding \\
\hline GO:0045211 & 19 & 18 & 19 & 56 & 63 & $7.76 \mathrm{E}-05$ & postsynaptic membrane \\
\hline GO:0008234 & 13 & 15 & 7 & 35 & 37 & $8.46 \mathrm{E}-05$ & cysteine-type peptidase activity \\
\hline
\end{tabular}




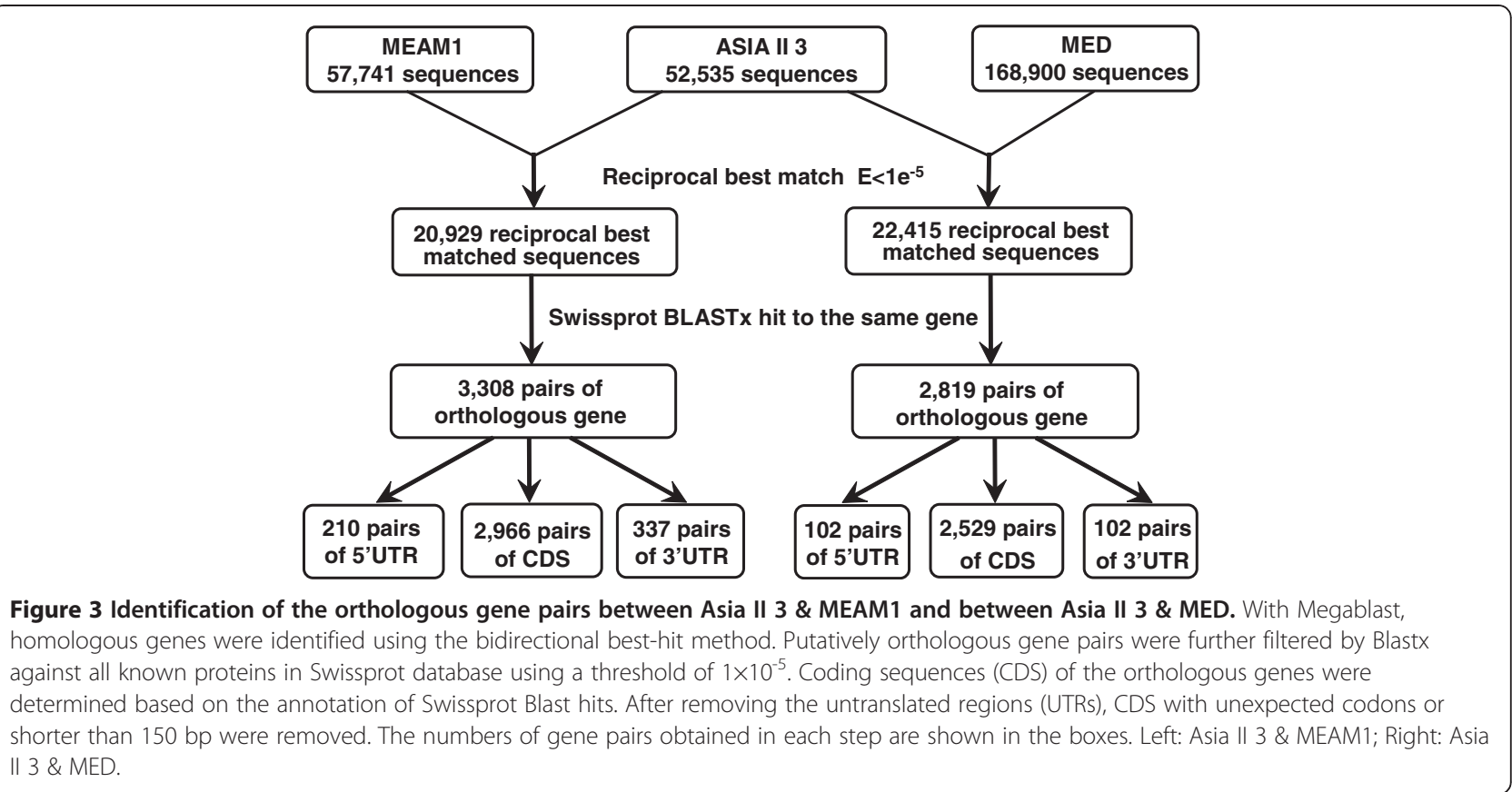

between MEAM1 and MED (0.83\%) (Table 4) [30]. Genome sequences are very useful for constructing phylogenetic trees with high resolution and accuracy. Using all the orthologous genes among the three species, a neighbor-joining tree was reconstructed (Figure 4). The phylogenetic distance between MEAM1 and MED is less than that of the invasive whiteflies to the native Asia II 3. This is not surprising as both MEAM1 and MED originated from the Middle East Asia Minor and Mediterranean Basin regions whereas Asia II 3 is a native species in China [14,34].

The sequence divergence between Asia II 3 and MEAM1 To reveal the molecular variation between Asia II 3 and MEAM1, the sequence divergence was analyzed between the orthologous genes of their transcriptomes (Table 5). The average divergence between the 5'UTRs of Asia II 3 and the MEAM1 orthologous genes is $3.35 \%$ which is

Table 4 Sequence divergence of MEAM1/Asia II 3 and MED/Asia II 3

\begin{tabular}{lll}
\hline & MEAM1/Asia II 3 & MED/Asia II 3 \\
\hline Total ortholog pairs: & 2,966 & 2,529 \\
\hline Total aligned length (kb): & $1,434.4$ & $1,072.1$ \\
\hline Mean aligned length (bp): & 483.6 & 423.9 \\
\hline Longest aligned length (bp): & 3,081 & 2,919 \\
\hline Mean homology (\%): & $98.27 \%$ & $98.16 \%$ \\
\hline Lowest homology (\%): & $70.43 \%$ & $79.68 \%$ \\
\hline Highest homology (\%): & $100 \%$ & $100 \%$ \\
\hline Standard deviation: & 0.00024 & 0.00029 \\
\hline
\end{tabular}

almost twice as much as between the same region of MEAM1 and MED. Similar to previous reports, the divergence rate at the CpG sites in the 5 'UTR $(8.28 \%)$ is much higher than that of non-CpG sites (3.05\%). For the 3'UTR, the overall difference between Asia II 3 and MEAM1 is $2.91 \%$. CpG and non-CpG sites differ at $12.42 \%$ and $2.52 \%$, respectively (Table 5 ). Hence, in the 3'UTR, CpG sites contain 4.93 times of differences compared to non-CpG sites. Among the 2,966 orthologous gene pairs, the overall divergence in CDS is $1.73 \%$. In non-CpG sites, the divergence is slightly lower (1.29\%), whereas the CpG site divergence (7.77\%) is about 6.0 times as high as the non-CpG site divergence (Table 5). Nucleotides in CDS can further be classified as nondegenerative (nd) sites (any nucleotide change cause amino acid replacement) and four fold degenerate (4d) sites (no substitution cause amino acid change). At nd sites the overall divergence is $0.47 \%$, whereas the overall

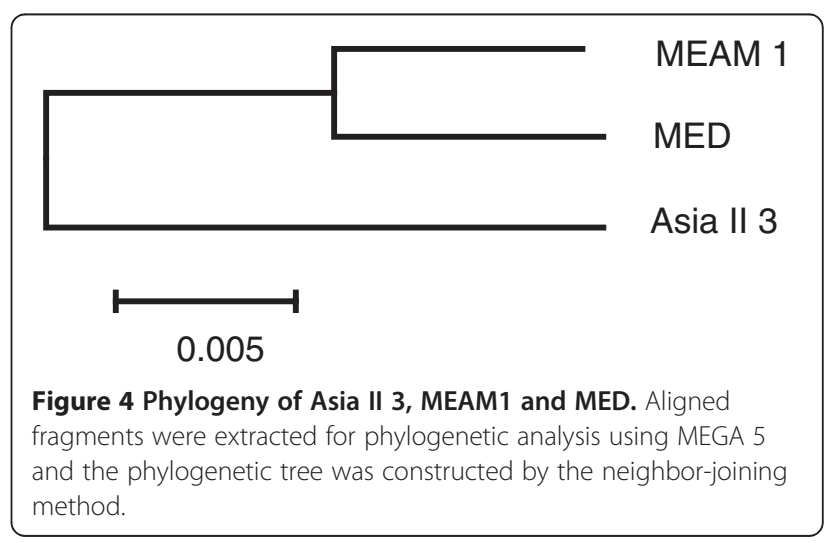


Table 5 Sequence divergence between MEAM1 and Asia II 3

\begin{tabular}{|c|c|c|c|c|c|c|c|}
\hline \multicolumn{8}{|c|}{ \% Differences } \\
\hline & $\%$ CpG & $\%$ GC & Loci & mean & $\mathrm{SE}$ & Compared kb & $\mathrm{ts} / \mathrm{tv}^{\mathrm{e}}$ \\
\hline $5^{\prime}$ UTRs $^{a}$ & 7.33 & 37.36 & 210 & & & & \\
\hline All & & & & 3.35 & 0.21 & 22.11 & 1.47 \\
\hline No CpG & & & & 3.05 & 0.21 & 20.49 & 1.34 \\
\hline CpG & & & & 8.28 & 1.03 & 1.62 & 2.38 \\
\hline $\mathrm{CDS}^{\mathrm{b}}$ & 7.13 & 43.44 & 2966 & & & & \\
\hline All & & & & 1.73 & 0.02 & 1434.41 & 2.94 \\
\hline No $\mathrm{CpG}$ & & & & 1.29 & 0.02 & 1332.11 & 2.53 \\
\hline $\mathrm{CpG}$ & & & & 7.77 & 0.12 & 102.30 & 4.17 \\
\hline nd sites ${ }^{c}$ & 6.27 & 44.17 & 2966 & & & & \\
\hline All & & & & 0.47 & 0.02 & 840.49 & 1.43 \\
\hline No $\mathrm{CpG}$ & & & & 0.42 & 0.02 & 787.78 & 1.29 \\
\hline $\mathrm{CpG}$ & & & & 1.27 & 0.07 & 52.71 & 2.41 \\
\hline $4 d$ sites $^{d}$ & 12.07 & 37.64 & 2966 & & & & \\
\hline All & & & & 5.30 & 0.07 & 200.59 & 2.18 \\
\hline No $\mathrm{CpG}$ & & & & 3.43 & 0.06 & 176.37 & 1.55 \\
\hline CpG & & & & 20.11 & 0.36 & 24.22 & 3.52 \\
\hline 3' UTRs & 4.68 & 35.74 & 337 & & & & \\
\hline All & & & & 2.91 & 0.16 & 46.95 & 2.01 \\
\hline No $\mathrm{CpG}$ & & & & 2.52 & 0.15 & 44.75 & 1.68 \\
\hline CpG & & & & 12.42 & 0.97 & 2.20 & 4.48 \\
\hline 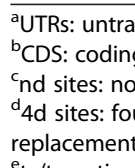 & $\begin{array}{l}\text { nslated reg } \\
\text { g sequence } \\
\text { n-degener } \\
\text { urfold-dege } \\
\text { t. }\end{array}$ & $\begin{array}{l}\text { gions. } \\
\text { e. } \\
\text { rative site } \\
\text { enerate s }\end{array}$ & $\begin{array}{l}\text { s. } \\
\text { sites wh }\end{array}$ & ere noc & 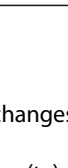 & s cause any amino & \\
\hline
\end{tabular}

divergence at $4 \mathrm{~d}$ sites $(5.3 \%)$ is 11.3 times of that at the nd sites. This is due to the fact that any nucleotide substitutions at an nd site will produce an amino acid change; therefore nd sites evolve under extensive functional constraints. To understand the mechanism of gene evolution in the B. tabaci complex, we also compared the ratio of transition (ts) and transversion (tv) of the three species [35]. Overall, the transitional differences are more frequent than transversional differences in 5'UTRs (1.47), CDS (2.94) and 3'UTRs (2.01) (Table 5). Interestingly, for all regions, the transition-transversion ratio is higher in the $\mathrm{CpG}$ positions than the non-CpG positions (Table 5). This is consistent with the suggestion that, in the CpG sites, the predominant type of mutations is cytosine deamination, which results in transitional differences [36].

The sequence divergence between Asia II 3 and MED For Asia II 3 and MED, the average differences between the 5'UTRs, CDS and the 3'UTRs are 3.57\%, 1.84\% and $3.13 \%$, respectively (Additional file 6). The sequence divergences are almost the same between Asia II 3 and
MEAM1 and nearly twice of the divergence between MEAM1 and MED which is $1.66 \%, 0.83 \%$ and $1.43 \%$ (Figure 5) [30]. Likewise, in the $4 \mathrm{~d}$ and nd regions, the same phenomenon was observed (Figure 5 and Additional file 6). It confirmed the previous analysis that the phylogenetic distance between MEAM1 and MED is shorter than that of the invasive whiteflies to the native Asia II 3 (Figure 4) [2,16]. These results indicate that despite high-sequence identity in their coding sequences, the Asia II 3, MEAM1 and MED B. tabaci species have diverged substantially between their transcriptomes. The levels of sequence divergence provide further supports to the previous proposition that Asia II 3, MEAM1 and MED are different species [25,26,37].

\section{Analysis of synonymous and non-synonymous sites}

The nonsynonymous (Ka) and synonymous (Ks) substitution rates have been widely used to measure the intensity of gene evolution. To identify genes undergoing purifying or positive selections, we estimated $\mathrm{Ka}$ and $\mathrm{Ks}$ rates of orthologous gene pairs. Among the 2,966 pairs of CDS between Asia II 3 and MEAM1, both a Ka and a Ks rate could be calculated for 1,373 orthologs (Additional file 7). The 1,373 sequence pairs had mean values of $\mathrm{Ka}, \mathrm{Ks}$ and $\mathrm{Ka} / \mathrm{Ks}$ of $0.0094,0.0623$ and 0.198 . These ratios are similar to the average $\mathrm{Ka} / \mathrm{Ks}$ ratio of MEAM1 \& MED (0.225), $\mathrm{Ka} / \mathrm{Ks}$ ratio of rat \& mouse (0.19) and human \& chimpanzee $(0.22)[30,38,39]$. The distribution of the $\mathrm{Ka} / \mathrm{Ks}$ ratio showed that the majority of genes (98.2\%, 1348/1373) have $\mathrm{Ka} / \mathrm{Ks}$ ratios less than 1, indicating the strong purifying selection for these genes. In addition, Fisher's exact test reports that nearly $56 \%$ of the genes are statistically significant $(P<0.01)$ (Additional

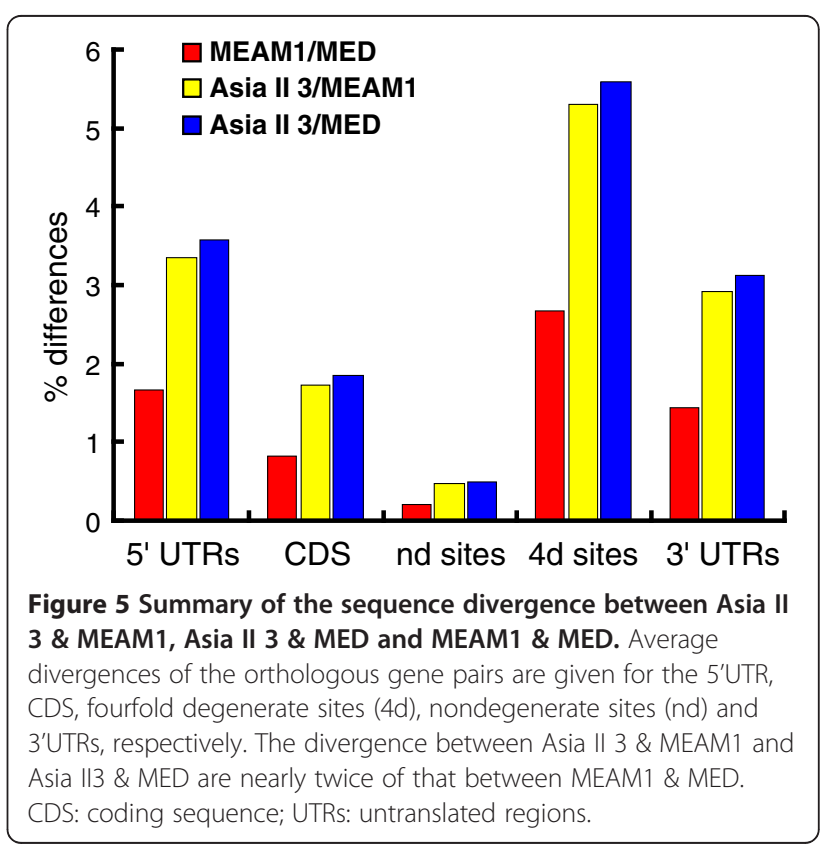


file 7). In this set of data, 25 orthologous gene pairs had a $\mathrm{Ka} / \mathrm{Ks}$ value $>1$ suggesting that strong positive selection acts on these genes. Among the sequences with $\mathrm{Ka} /$ Ks values $>1$, a number of genes are involved in protein metabolism, such as peptide deformylase, cathepsin, cysteine proteinase and metalloendopeptidase, suggesting this process is critical for the differentiation of the $B$. tabaci species complex. Between Asia II 3 and MED, a total of 1,221 orthologs had $\mathrm{Ka}$ and $\mathrm{Ks}$ rates and the mean values of $\mathrm{Ka}, \mathrm{Ks}$ and $\mathrm{Ka} / \mathrm{Ks}$ were 0.0092 , 0.0729 and 0.201 . The list of Asia II 3 and MED genes with $\mathrm{Ka} /$ $\mathrm{Ks}$ and functional annotation is presented in Additional file 8 . The distribution of $\mathrm{Ka} / \mathrm{Ks}$ between Asia II 3 and MED is similar to that of Asia II 3 and MEAM1, in which $7.9 \%$ of the genes had a high $\mathrm{Ka} / \mathrm{Ks}>0.5,44.8 \%$ of the genes were highly conserved $(\mathrm{Ka} / \mathrm{Ks}<0.1)$ and 25 genes had a $\mathrm{Ka} / \mathrm{Ks}>1$. Even though a number of genes under positive selection have been identified in our study, the simple $\mathrm{Ka} / \mathrm{Ks}$ calculations are quite conservative and may fail to detect positive selection even when it exists [40]. Further studies using more sophisticated site and branch specific models are needed for estimating $\mathrm{Ka} / \mathrm{Ks}$.

\section{Sequences with very weak amino-acid similarity}

The 2,966 sequence pairs between Asia II 3 and MEAM1 had a mean homology of $98.27 \%$ and the homology for individual gene pair ranged from $70.43 \%$ to $100 \%$ (Additional file 5). Among these sequence pairs, only 94 show $100 \%$ homology (Additional file 5 ). This is much lower compared to the 604 identical sequences between MEAM1 and MED [30]. These results further suggest that the divergence between Asia II 3 and MEAM1 is much higher that that between MEAM1 and MED (Figure 4). To reveal the proteins responsible for the differences between the two species, the functions of sequence pairs with weak amino-acid similarity were analyzed. Interestingly, many of the divergent genes were related to protein kinase and metabolism, such as Protein kinase C (89.39\%), Serine/threonine-protein kinase (90.42\%), glutathione S-transferase (92.59\%) and ethanolaminephosphotransferase (92.75\%) (Additional file 5). To gain further insights into the diverged sequences, we compiled sequence identify data into gene functional groups using the Kyoto Encyclopedia of Genes and Genomes (KEGG) classifications. To prevent false positive results, only those classes containing five or more entries were shown (Additional file 9). Interestingly, we found that several classes of genes involved in signal transduction were highly conserved between the two species, such as phototransduction, taste transduction, olfactory transduction and MAPK signaling pathway (Additional file 9). On the other hand, the most divergent KEGG classes were about metabolism, such as Metabolism of xenobiotics by cytochrome P450, Glutathione metabolism, and Ascorbate and aldarate metabolism (Table 6). It suggests that the divergence of cytochrome $\mathrm{P} 450$ genes in the metabolism of xenobiotics might be important for the differentiation between MEAM1 and Asia II 3 and warrant further investigation.

Table 6 Mean identity of the orthologous gene pairs by KEGG classification

\begin{tabular}{|c|c|c|c|}
\hline Description & Pathway & Number of genes & Average identity \\
\hline Metabolism of xenobiotics by cytochrome P450 & ko00980 & 17 & $96.99 \%$ \\
\hline Drug metabolism - cytochrome P450 & ko00982 & 17 & $96.99 \%$ \\
\hline Glutathione metabolism & ko00480 & 18 & $97.59 \%$ \\
\hline Ascorbate and aldarate metabolism & ko00053 & 5 & $97.71 \%$ \\
\hline Oxidative phosphorylation & ko00190 & 41 & $97.84 \%$ \\
\hline Lysosome & ko04142 & 46 & $97.90 \%$ \\
\hline Pentose and glucuronate interconversions & ko00040 & 11 & $98.14 \%$ \\
\hline Ribosome & ko03010 & 14 & $98.18 \%$ \\
\hline Ubiquinone and terpenoid-quinone biosynthesis & ko00130 & 7 & $98.19 \%$ \\
\hline Glyoxylate and dicarboxylate metabolism & ko00630 & 6 & $98.20 \%$ \\
\hline Other types of O-glycan biosynthesis & ko00514 & 9 & $98.33 \%$ \\
\hline Steroid hormone biosynthesis & ko00140 & 15 & $98.36 \%$ \\
\hline MAPK signaling pathway - yeast & ko04011 & 6 & $98.37 \%$ \\
\hline Cardiac muscle contraction & ko04260 & 10 & $98.39 \%$ \\
\hline Insect hormone biosynthesis & ko00981 & 5 & $98.40 \%$ \\
\hline Cytokine-cytokine receptor interaction & ko04060 & 8 & $98.50 \%$ \\
\hline Glycosaminoglycan degradation & ko00531 & 7 & $98.51 \%$ \\
\hline Retinol metabolism & ko00830 & 19 & $98.54 \%$ \\
\hline
\end{tabular}




\section{Analysis of SNP}

To further understand the mechanism of divergence, we analyzed the potential SNP sites in CDS of the orthologous gene pairs between Asia II 3 and MEAM1. For Asia II 3, a total of 138 SNPs were identified within the $1433.63 \mathrm{~kb}$ aligned regions, about 1 every ten thousands bp. The complete list of SNPs with annotation can be found in Additional file 10. Of the 138 SNPs, 96 (69.6\%) were synonymous and $42(30.4 \%)$ were non-synonymous. This percentage of SNPs is much lower than those obtained in previous analyses in other insects [41,42]. The possible reason is that our B. tabaci populations were established from a pair of B. tabaci and the samples were collected within five generations. For MEAM1, a slightly higher number of SNPs (248, about 1.7 every ten thousands bp) were found in the orthologous gene pairs with 196 synonymous and 52 non-synonymous (Additional file 10). Compared with the average divergence between Asia II 3 and MEAM1 in CDS (1.83\%), the percentage of SNPs is more than 100 times lower. Next, the potential SNP sites in the CDS of orthologous gene pairs between Asia II 3 and MED were analyzed. Our results showed that a total of 56 SNPs in Asia II 3 and 627 SNPs in MED were identified within the 1,071 $\mathrm{kb}$ aligned regions (Additional file 11). The large variation between the numbers of SNPs in Asia II 3 and MED is probably due to the difference in the sequencing among of Asia II 3 (1G) and MED (3G) (Additional file 1). Some of the SNPs in Asia II 3 might have been filtered out because only SNP sites with the minimum read depth of 10 reads were selected. Thses results are consistent with previous findings, in which overall number of SNPs decreases at lower coverage levels [43]. While the SNPs we have identified here are suitable for future research, more rigorous statistical tests are required to confirm the current results as well as to detect specific codons undergoing adaptive changes. In addition, further studies of the SNPs on population samples are warranted as our data were generated from inbred lab colonies.

\section{Conclusions}

In summary, this study dramatically increases the number of genes from the native Asia II $3 \mathrm{~B}$. tabaci species. Together with the previously available MEAM1 and MED transcriptomes, this study is the first globally comparative analyses of the genetic differences between the invasive and indigenous $B$. tabaci species. Based on sequence homology, a group of 3,023 protein families conserved among the Asia II 3, MEAM1 and MED species were identified. These protein families might be responsible for core cellular and physiological functions of the B. tabaci complex. Sequence comparisons of all orthologous gene pairs revealed that the average genetic divergences between Asia II 3 and invasive MEAM1 are nearly twice of that between MEAM1 and MED, in accordance with previous genetic studies. The divergent genes identified in this study will be an invaluable resource to reveal the possible mechanisms of $B$. tabaci invasion, displacement and speciation.

\section{Methods}

\section{Insect rearing}

Stock culture of the Asia II 3 (mitochondrial cytochrome oxidase I gene GenBank accession no: AJ867556) was maintained on cotton, Gossypium hirsutum (Malvaceae) cv. Zhe-Mian 1793 in a climate chamber (see conditions below). The purity of the culture was monitored using the random amplified polymorphic DNA-PCR technique with the primer H16 (5'-TCTCAGCTGG-3') [44]. For sample preparation, a pair of virgin adults of $B$. tabaci Asia II 3 were released onto a cotton plant to oviposit and develop for five generations at $27 \pm 1^{\circ} \mathrm{C}$, a photoperiod of $14 \mathrm{~h}$ light: $10 \mathrm{~h}$ darkness and $70 \pm 10 \%$ relative humidity [45]. The same protocols were used to raise the MEAM1 and MED whiteflies for sample collection and subsequent transcriptome data generation.

\section{Sample preparation and RNA isolation}

In order to get an overall picture of the Asia II 3 whitefly transcriptome, we collected the samples from four different developing stages: 1) egg \& nymph (the eggs are extremely small, therefore a mixture of eggs and first to third instar nymphs were collected as one sample); 2) pupa; 3) female adult and 4) male adult. To ensure that the whitefly adults are in the same developmental stage, only newly emerged adults were collected. Previously, samples from MEAM1 and MED have been collected using the same strategy $[29,30]$. Total RNA was isolated from the four samples using SV total RNA isolation system (Promega) according to the manufacturer's protocol [46]. RNA integrity was confirmed using the 2100 Bioanalyzer (Agilent Technologies) with a minimum RNA integrated number value of 8 . Then, equal amount of RNA from egg \& nymph, pupa, female adult and male adult were mixed, and mRNA was purified from the mixture using oligo $(\mathrm{dT})$ magnetic beads.

\section{Library preparation and Illumina sequencing}

For transcriptome sequencing, a 200 bp cDNA library was prepared using Illumina's kit as previously described [29]. The library was not normalized because we intend to use the resulting sequence reads to analyze the level of gene expression. The cDNA library was sequenced for both ends on the GAII Illumina sequencing platform (a single lane) at The Beijing Genome Institute (Shenzhen, China). The total sequencing amount was 1G. The raw reads were filtered by removing adaptor sequences, 
empty reads and low quality sequences (reads with unknown sequences 'N') [30]. Next, the reads were randomly clipped into $21 \mathrm{bp} \mathrm{K}$-mers for assembly using SOAPdenovo software because the 21-mer provided the best result for transcriptome assembly. Small K-mers made the graph very complex; while large K-mers have poor overlap in low sequencing depth regions [31]. For alleles, the nucleotides with the highest frequency were selected. The resultant contigs were joined into scaffolds based on the mate pairs information and the scaffolds were clustered using TGI Clustering tools [47]. Assembled genes were used for subsequent analyses and are referred to as "distinct sequences".

\section{Data deposit}

The data sets of Illumina sequencing are available at the NCBI Short Read Archive (SRA) database with the accession number: SRR062575. The assembled sequences were deposited in the NCBI Transcriptome Shotgun Assembly (TSA) database under the accession number of HP777244 to HP823074 and can be searched using the GeneID listed in Additional file 2, Supporting information.

\section{Functional annotation and gene expression analysis}

Distinct sequences were annotated by Blast search against the NCBI nr database with a cut-off E-value of $10^{-5}$. GO annotation was analyzed by Blast2GO software [48]. The GO terms were retrieved from Blast hits with an E-value threshold of $10^{-5}$. Comparisons of the distribution of GO terms among the Asia II, MEAM1 and MED transcriptomes were done using the Web Gene Ontology Annotation Plot (WEGO) [49]. Pathway annotation was performed using Blastall software against the KEGG database. Based on the number of reads for a gene, gene expression levels can be estimated from Illumina sequencing with great accuracy [50,51]. To estimate the level of gene expression, the number of reads mapped to each distinct sequence was extracted. Since read mapping is sensitive to the size of the target reference sequence and sequencing amount, we adjusted the raw read count by the total number of reads mapped and the length of the gene by calculating Reads Per Kilobase per Million mapped reads (RPKM) [50].

\section{Analysis of protein families}

To reveal the functional differences among Asia II 3, MEAM1 and MED transcriptomes, we analyzed their protein families. Using Blastx (E-value $<10^{-5}$ ), the translated region of each gene was identified by aligning the sequence to the Swissprot database. The longest translated protein sequence for each gene was then extracted and sequences less than 200 bp were removed. To identify protein families among the three transcriptomes, an all-against-all Blastp was performed for all the translated genes from the three transcriptomes. Blastp results were further analyzed by a Markov Cluster Algorithm (MCL) with an inflation factor of 1.6. The protein families belonging to all the three transcriptomes were referred to as "Core protein families". Based on the GO annotation of Asia II 3, MEAM1 and MED transcriptomes, we calculated the total number of genes under each GO term in the "Core protein families" and the three transcriptomes. For each GO term, its enrichment in the "Core protein families" was measured using the hypergeometric test with an cut-off $p$ value of $10^{-5}$ [33].

\section{Identification of orthologous genes and prediction of coding and untranslated regions}

The orthologous genes between Asia II 3 and MEAM1 and those between Asia II 3 and MED were identified respectively according to the previous description using MegaBLAST [30]. Briefly, pairs of sequences that were reciprocally a best hit and with a minimum length of $200 \mathrm{bp}$ were retained as putative orthologs. To remove potential paralogs, only pairs of sequences unambiguously mapped to the same protein in Swissprot database with an E-value $<1 \times 10^{-5}$ were selected. The CDS of the orthologous genes were determined by BLASTx the Swissprot database with an $E$ value $<1 \times 10^{-5}$. The start codon was determined by examination of the in-frame ATG codon of the aligned reference protein. The stop codon position was determined by examination of inframe TAA, TAG and TGA motifs present within $30 \mathrm{bp}$ of the stop codon of the reference protein. The 5'UTR and 3'UTR regions were defined based on the position of start codon, stop codon and predicted CDS. To prevent false positive results, only UTR pairs with an Evalue $<1 \times 10^{-30}$ were selected for further analyses. CDS containing unexpected stop codon(s) and shorter than $150 \mathrm{bp}$ were removed.

\section{Sequence divergence analyses and estimation of substitution rates}

The 5'UTR, CDS and 3'UTR regions were separately extracted from each pair of orthologs. The CDS and UTR regions were aligned separately to each other with a MegaBlast algorithm and checked manually for errors. Only the homologous regions of each gene pair were extracted for sequence comparison. Sequence divergence between the homologous regions of each gene pair was calculated by dividing the number of substitutions by the number of base pairs compared. The average divergence between transcriptomes was determined by dividing the total number of substitutions by the total number of base pairs compared. The sequence divergence at nondegenerate (nd), fourfold degenerate (4d), $\mathrm{CpG}$ and non-CpG regions was determined respectively according to the previous descriptions [39]. The ratio of 
transition over transversion (ts/tv) was determined for the 5'UTR, CDS and 3'UTR as well. Using the KaKs Calculator, we also estimated the substitution rates for nonsynonymous sites (Ka) and synonymous (Ks) sites (YN method) [52,53].

\section{Phylogeny of Asia II 3, MEAM1 and MED}

The orthologous genes among Asia II 3, MEAM1 and MED were selected for sequence alignment using MUSCLE [54] and the aligned fragments were extracted for phylogenetic analysis using MEGA 5 [55]. The evolutionary history was inferred using the neighbor-joining method [56]. All positions containing gaps and missing data were eliminated and the within population polymorphisms were not included for divergence estimation. The analysis involved a total of 686,101 positions in the final dataset. The evolutionary distances were computed using the Maximum Composite Likelihood method [57] and are in the units of the number of base substitutions per site.

\section{SNP analysis}

To reveal the mechanism of divergence between Asia II 3 and MEAM1, we analyzed the potential SNP sites in CDS of the orthologous gene pairs between the invasive and indigenous whitefly species. The orthologous gene pairs of Asia II 3 \& MEAM1 and Asia II 3 \& MED were subjected to SNP analysis according to the previous description with slight modifications [58]. In short, the Illumina sequencing reads were mapped to the orthologous CDS regions of each gene using TopHat (V1.2.0) with the following parameters: -g1 -r 200 -mate-std-dev 20 -I 10000 [59]. All possible SNP sites with the minimum read depth of 10 reads were then identified by SAMTools (V0.1.13) based on aligned outcomes [60]. The analyses of amino acid mutation and functional annotation were performed by a custom-written algorithm.

\section{Additional files}

Additional file 1: Summary of Asia II 3, MEAM1 and MED transcriptomes.

Additional file 2: Top BLASTx hits from NCBI nr database. BLASTx results against the NCBI nr database for all the distinct sequences with a cut-off E value above 1.0E-5 are shown. BT: Bemisia tabaci.

Additional file 3: Gene Ontology comparison of Asia II 3, MEAM1 and MED transcriptomes. The Gene Ontology (GO) terms are summarized in three main categories: biological process, cellular component and molecular function. The left $y$-axis indicates the percentage of genes within a specific category in that main category. The right $y$-axis means the number of genes in a category. A. GO comparison between Asia $\| 3$ and MEAM1. B. GO comparison between Asia $\| 3$ and MED.

Additional file 4: Gene expression level of Asia II 3 transcriptome. The length of each distinct sequence and numbers of raw reads for each gene are listed. Swissprot and nr annotations were shown as well. RPKM: reads per kilobase per million mapped reads.
Additional file 5: List of the orthologous gene pairs between Asia II 3 and MEAM1. The length of coding sequence, homology and Swissprot, nr annotations are shown.

Additional file 6: Sequence divergence between Asia II 3 and MED transcriptomes.

Additional file 7: Ka and Ks of each orthologous gene pairs between Asia II 3 and MEAM1. Ka: nonsynonymous substitution rate; Ks: synonymous substitution rate.

Additional file 8: Ka and Ks of each orthologous gene pairs between Asia II 3 and MED. Ka: nonsynonymous substitution rate; Ks: synonymous substitution rate.

Additional file 9: Mean sequence identity by KEGG classification. The KEGG pathway description and ID are shown. Number of sequences: total number of sequences in a specific KEGG pathway.

Additional file 10: SNP sites in the coding sequence of orthologous gene pairs between Asia II 3 and MEAM1.

Additional file 11: SNP sites in the coding sequence of orthologous gene pairs between Asia II 3 and MED.

\section{Competing interests}

The authors declare that they have no competing interests.

\section{Authors' contributions}

XWW, QYZ, JBL and SSL conceived and designed the experimental plan. XWW, QYZ, JBL and GHY performed experiments. XWW, QYZ and YJW analyzed and interpreted the sequence data. XWW and SSL drafted the manuscript. All authors read and approved the final manuscript.

\section{Acknowledgements}

Financial support for this study was provided by the National Basic Research Program of China (Project 2009CB119203), and the National Natural Science Foundation of China (Project 31021003, 31071686). We thank Laura Boykin for editing the manuscript.

\section{Author details}

${ }^{1}$ Ministry of Agriculture Key Laboratory of Agricultural Entomology, Institute of Insect Sciences, Zhejiang University, 866 Yuhangtang Road, Hangzhou 310058, China. ${ }^{2}$ The University of Queensland, Queensland Brain Institute, Brisbane, Qld 4072, Australia.

Received: 19 April 2012 Accepted: 28 September 2012 Published: 4 October 2012

\section{References}

1. Dinsdale A, Cook L, Riginos C, Buckley Y, De Barro PJ: Refined global analysis of Bemisia tabaci (Gennadius) (Hemiptera: Sternorrhyncha: Aleyrodoidea) mitochondrial CO1 to identify species level genetic boundaries. Ann Entomol Soc Am 2010, 103:196-208.

2. De Barro PJ, Liu SS, Boykin LM, Dinsdale AB: Bemisia tabaci: a statement of species status. Annu Rev Entomol 2011, 56:1-19.

3. Hu J, De Barro PJ, Zhao H, Wang J, Nardi F, Liu SS: An extensive field survey combined with a phylogenetic analysis reveals rapid and widespread invasion of two alien whiteflies in china. PLoS One 2011 6:e16061.

4. Liu SS, Colvin J, De Barro PJ: Species concepts as applied to the whitefly Bemisia tabaci systematics: how many species are there? J Integr Agri 2012, 11:176-186.

5. Alemandri V, De Barro P, Bejerman N, Arguello Caro EB, Dumon AD, Mattio MF, Rodriguez SM, Truoli G: Species within the Bemisia tabaci (Hemiptera: Aleyrodidae) complex in soybean and bean crops in Argentina. J Econ Entomol 2012, 105:48-53.

6. Parrella G, Scassillo L, Giorgini M: Evidence for a new genetic variant in the Bemisia tabaci species complex and the prevalence of the biotype Q in southern Italy. J Pest Sci 2012, 85:227-238.

7. Brown JK: Molecular markers for the identification and global tracking of whitefly vector-Begomovirus complexes. Virus Res 2000, 71:233-260. 
8. Liu SS, De Barro PJ, Xu J, Luan JB, Zang LS, Ruan YM, Wan FH: Asymmetric mating interactions drive widespread invasion and displacement in a whitefly. Science 2007, 318:1769-1772

9. Jiu M, Zhou XP, Tong L, Xu J, Yang X, Wan FH, Liu SS: Vector-virus mutualism accelerates population increase of an invasive whitefly. PLOS One 2007, 2:e182.

10. Crowder DW, Horowitz AR, De Barro PJ: Mating behaviour, life history and adaptation to insecticides determine species exclusion between whiteflies. J Anim Ecol 2010, 79:563-570.

11. Gorman K, Slater R, Blande JD, Clarke A, Wren J, McCaffery A, Denholm I: Cross-resistance relationships between neonicotinoids and pymetrozine in Bemisia tabaci (Hemiptera: Aleyrodidae). Pest Manag Sci 2010, 66:1186-1190.

12. Czosnek H, Ghanim M, Ghanim M: The circulative pathway of begomoviruses in the whitefly vector Bemisia tabaci; insights from studies with Tomato yellow leaf curl virus. Ann Appl Biol 2002, 140:215-231.

13. Dalton R: Whitefly infestations: the Christmas Invasion. Nature 2006, 443:898-900

14. Naranjo SE, Castle SJ, De Barro PJ, Liu SS: Population Dynamics, Demography, Dispersal and Spread of Bemisia tabaci. In Bemisia: Bionomics and Management of a Global Pest. Edited by Stansly PA, Naranjo SE. Heidelberg: Springer; 2010:185-226.

15. Brown JK, Frohlich DR, Rosell RC: The sweetpotato or silverleaf whiteflies: biotypes of Bemisia tabaci or a species complex? Annu Rev Entomol 1995 40:511-534.

16. Boykin LM, Shatters RG, Rosell RC, Mckenzie CL, Bagnall RA, De Barro PJ, Frohlich DR: Global relationships of Bemisia tabaci (Hemiptera: Aleyrodidae) revealed using Bayesian analysis of mitochondrial COI DNA sequences. Mol Phylogenet Evol 2007, 44:1306-1319.

17. Luan JB, XU J, Lin KK, Zalucki MP, Shu-sheng L: Species exclusion between an invasive and an indigenous whitefly on host plants with differential levels of suitability. J Integr Agri 2012, 11:215-224.

18. Muñiz M, Nombela G: Differential variation in development of the B- and Q-biotypes of Bemisia tabaci (Homoptera: Aleyrodidae) on sweet pepper at constant temperatures. Environ Entomol 2001, 30:720-727.

19. Nauen R, Stumpf N, Elbert A: Toxicological and mechanistic studies on neonicotinoid cross resistance in Q-type Bemisia tabaci (Hemiptera: Aleyrodidae). Pest Manag Sci 2002, 58:868-875.

20. Horowitz AR, Kontsedalov S, Khasdan V, Ishaaya I: Biotypes B and Q of Bemisia tabaci and their relevance to neonicotinoid and pyriproxyfen resistance. Arch Insect Biochem Physiol 2005, 58:216-225.

21. Fernandez E, Gravalos C, Haro PJ, Cifuentes D, Bielza P: Insecticide resistance status of Bemisia tabaci Q-biotype in south-eastern Spain. Pest Manag Sci 2009, 65:885-891.

22. Ghanim M, Kontsedalov S: Gene expression in pyriproxyfen-resistant Bemisia tabaci Q biotype. Pest Manag Sci 2007, 63:776-783.

23. Stewart CNJ: Weedy and Invasive Plant Genomics. New Jersey: WileyBlackwell; 2009.

24. Charlesworth D, Charlesworth B, McVean GA: Genome sequences and evolutionary biology, a two-way interaction. Trends Ecol Evol 2001, $16: 235-242$

25. Wang P, Sun DB, Qiu BL, Liu SS: The presence of six putative species of the whitefly Bemisia tabaci complex in China as revealed by crossing experiments. Insect Sci 2011, 18:67-77.

26. Sun DB, Xu J, Luan JB, Liu SS: Reproductive incompatibility between the $B$ and $\mathrm{Q}$ biotypes of the whitefly Bemisia tabaci: genetic and behavioural evidence. Bull Entomol Res 2011, 101:211-220.

27. Moya A, Guirao P, Cifuentes D, Beitia F, Cenis JL: Genetic diversity of Iberian populations of Bemisia tabaci (Hemiptera: Aleyrodidae) based on random amplified polymorphic DNA-polymerase chain reaction. $\mathrm{Mol}$ Ecol 2001, 10:891-897.

28. Elbaz M, Weiser M, Morin S: Asymmetry in thermal tolerance trade-offs between the $\mathrm{B}$ and $\mathrm{Q}$ sibling species of Bemisia tabaci (Hemiptera: Aleyrodidae). J Evol Biol 2011, 24:1099-1109.

29. Wang XW, Luan JB, Li JM, Bao YY, Zhang CX, Liu SS: De novo characterization of a whitefly transcriptome and analysis of its gene expression during development. BMC Genomics 2010, 11:400.

30. Wang XW, Luan JB, Li JM, Su YL, Xia J, Liu SS: Transcriptome analysis and comparison reveal divergence between two invasive whitefly cryptic species. BMC Genomics 2011, 12:458.

31. Li R, Zhu H, Ruan J, Qian W, Fang X, Shi Z, Li Y, Li S, Shan G, Kristiansen K, Li S, Yang H, Wang J, Wang J: De novo assembly of human genomes with massively parallel short read sequencing. Genome Res 2010, 20:265-272.
32. Elmer KR, Fan S, Gunter HM, Jones JC, Boekhoff S, Kuraku S, Meyer A: Rapid evolution and selection inferred from the transcriptomes of sympatric crater lake cichlid fishes. Mol Ecol 2010, 19(Suppl 1):197-211.

33. Yin Y, Martin J, Abubucker S, Scott AL, McCarter JP, Wilson RK, Jasmer DP, Mitreva M: Intestinal transcriptomes of nematodes: comparison of the parasites Ascaris suum and Haemonchus contortus with the free-living Caenorhabditis elegans. PLoS Negl Trop Dis 2008, 2:e269.

34. Zang LS, Chen WQ, Liu SS: Comparison of performance on different host plants between the B biotype and a non-B biotype of Bemisia tabaci from Zhejiang, China. Entomol Exp Appl 2006, 121:221-227.

35. Yang Z, Yoder AD: Estimation of the transition/transversion rate bias and species sampling. J Mol Evol 1999, 48:274-283.

36. Shen JC, Rideout WM 3rd, Jones PA: The rate of hydrolytic deamination of 5methylcytosine in double-stranded DNA. Nucleic Acids Res 1994, 22:972-976.

37. Xu J, De Barro PJ, Liu SS: Reproductive incompatibility among genetic groups of Bemisia tabaci supports the proposition that the whitefly is a cryptic species complex. Bull Entomol Res 2010, 100:359-366.

38. Makalowski W, Boguski MS: Evolutionary parameters of the transcribed mammalian genome: an analysis of 2,820 orthologous rodent and human sequences. Proc Nat Acad Sci USA 1998, 95:9407-9412.

39. Hellmann I, Zollner S, Enard W, Ebersberger I, Nickel B, Paabo S: Selection on human genes as revealed by comparisons to chimpanzee cDNA Genome Res 2003, 13:831-837.

40. Nielsen R, Yang Z: Likelihood models for detecting positively selected amino acid sites and applications to the HIV-1 envelope gene. Genetics 1998, 148:929-936.

41. Wondji CS, Hemingway J, Ranson H: Identification and analysis of single nucleotide polymorphisms (SNPs) in the mosquito Anopheles funestus, malaria vector. BMC Genomics 2007, 8:5

42. Morlais I, Poncon N, Simard F, Cohuet A, Fontenille D: Intraspecific nucleotide variation in Anopheles gambiae: new insights into the biology of malaria vectors. Am J Trop Med Hyg 2004, 71:795-802.

43. Kim SY, Lohmueller KE, Albrechtsen A, Li Y, Korneliussen T, Tian G, Grarup N, Jiang $T$, Andersen $G$, Witte $D$, Jorgensen $T$, Hansen $T$, Pedersen $O$, Wang J, Nielsen R: Estimation of allele frequency and association mapping using next-generation sequencing data. BMC Bioinformatics 2011, 12:231.

44. Luo C, Jones CM, Devine G, Zhang F, Denholm I, Gorman K: Insecticide resistance in Bemisia tabaci biotype $\mathrm{Q}$ (Hemiptera: Aleyrodidae) from China. Crop Prot 2010, 29:429-434.

45. Li FF, Xia J, Li JM, Liu SS, Wang XW: p38 MAPK is a component of the signal transduction pathway triggering cold stress response in the MED cryptic species of Bemisia tabaci. J Integr Agri 2012, 11:302-311.

46. Li JM, Ruan YM, Li FF, Liu SS, Wang XW: Gene expression profiling of the whitefly (Bemisia tabaci ) Middle East - Asia Minor 1 feeding on healthy and Tomato yellow leaf curl China virus-infected tobacco. Insect Sci 2011, 18:11-22.

47. Pertea G, Huang X, Liang F, Antonescu V, Sultana R, Karamycheva S, Lee $Y$, White J, Cheung F, Parvizi B, Tsai J, Quackenbush J: TIGR Gene Indices clustering tools (TGICL): a software system for fast clustering of large EST datasets. Bioinformatics 2003, 19:651-652.

48. Conesa A, Gotz S, Garcia-Gomez JM, Terol J, Talon M, Robles M: Blast2GO: a universal tool for annotation, visualization and analysis in functional genomics research. Bioinformatics 2005, 21:3674-3676.

49. Ye J, Fang L, Zheng H, Zhang Y, Chen J, Zhang Z, Wang J, Li S, Li R, Bolund L, Wang J: WEGO: a web tool for plotting GO annotations. Nucleic Acids Res 2006, 34:W293-W297.

50. Mortazavi A, Williams BA, McCue K, Schaeffer L, Wold B: Mapping and quantifying mammalian transcriptomes by RNA-Seq. Nat Methods 2008, 5:621-628.

51. Wolf JB, Bayer T, Haubold B, Schilhabel M, Rosenstiel P, Tautz D: Nucleotide divergence vs. gene expression differentiation: comparative transcriptome sequencing in natural isolates from the carrion crow and its hybrid zone with the hooded crow. Mol Ecol 2010, 19(Suppl 1):162-175.

52. Yang Z, Nielsen R: Estimating synonymous and nonsynonymous substitution rates under realistic evolutionary models. Mol Biol Evol 2000 17:32-43.

53. Zhang Z, Li J, Zhao XQ, Wang J, Wong GK, Yu J: KaKs_Calculator: calculating $\mathrm{Ka}$ and $\mathrm{Ks}$ through model selection and model averaging. Genomics Proteomics Bioinformatics 2006, 4:259-263.

54. Edgar RC: MUSCLE: multiple sequence alignment with high accuracy and high throughput. Nucleic Acids Res 2004, 32:1792-1797. 
55. Tamura K, Peterson D, Peterson N, Stecher G, Nei M, Kumar S: MEGA5: molecular evolutionary genetics analysis using maximum likelihood, evolutionary distance, and maximum parsimony methods. Mol Biol Evol 2011, 28:2731-2739.

56. Saitou N, Nei M: The neighbor-joining method: a new method for reconstructing phylogenetic trees. Mol Biol Evol 1987, 4:406-425.

57. Tamura K, Nei M, Kumar S: Prospects for inferring very large phylogenies by using the neighbor-joining method. Proc Natl Acad Sci USA 2004, 101:11030-11035.

58. You FM, Huo N, Deal KR, Gu YQ, Luo MC, McGuire PE, Dvorak J, Anderson OD: Annotation-based genome-wide SNP discovery in the large and complex Aegilops tauschii genome using next-generation sequencing without a reference genome sequence. BMC Genomics 2011, 12:59.

59. Trapnell C, Pachter L, Salzberg SL: TopHat: discovering splice junctions with RNA-Seq. Bioinformatics 2009, 25:1105-1111.

60. Li H, Handsaker B, Wysoker A, Fennell T, Ruan J, Homer N, Marth G, Abecasis G, Durbin R: The Sequence Alignment/Map format and SAMtools. Bioinformatics 2009, 25:2078-2079.

doi:10.1186/1471-2164-13-529

Cite this article as: Wang et al: Analysis of a native whitefly

transcriptome and its sequence divergence with two invasive whitefly species. BMC Genomics 2012 13:529.

\section{Submit your next manuscript to BioMed Central and take full advantage of:}

- Convenient online submission

- Thorough peer review

- No space constraints or color figure charges

- Immediate publication on acceptance

- Inclusion in PubMed, CAS, Scopus and Google Scholar

- Research which is freely available for redistribution 